\title{
Psychological Stress Induces Temporary Masticatory Muscle Mechanical Sensitivity in Rats
}

\author{
Fei Huang, Min Zhang, Yong-Jin Chen, Qiang Li, and An-Zhen Wu \\ Department of General Dentistry and Emergency, School of Stomatology, The Fourth Military Medical University, \\ Xi'an, Shaanxi 710032, China \\ Correspondence should be addressed to Min Zhang, zhangmin@fmmu.edu.cn and Yong-Jin Chen, cyj1229@fmmu.edu.cn
}

Received 15 September 2010; Revised 13 December 2010; Accepted 30 December 2010

Academic Editor: Monica Fedele

Copyright ( $\odot 2011$ Fei Huang et al. This is an open access article distributed under the Creative Commons Attribution License, which permits unrestricted use, distribution, and reproduction in any medium, provided the original work is properly cited.

\begin{abstract}
To explore the relationship between psychological stress and masticatory muscle pain, we created a communication stress animal model to determine whether psychological stress could induce increased mechanical sensitivity in masticatory muscles and to study the changes of mechanical nociceptive thresholds after stress removal. Forty-eight male Sprague-Dawley rats were divided into a control group (CON), a foot-shocked group (FS, including 3 subgroups recorded as FS-1, FS-2, and FS-3), a psychological stress group (PS), and a drug treatment group (DT). PS and DT rats were confined in a communication box for one hour a day to observe the psychological responses of neighboring FS rats.Measurements of the mechanical nociceptive thresholds of the bilateral temporal and masseter muscles showed a stimulus-response relationship between psychological stress and muscle mechanical sensitivity. The DT rats, who received a diazepam injection, showed almost the same mechanical sensitivity of the masticatory muscles to that of the control in response to psychological stress. Fourteen days after the psychological stressor was removed, the mechanical nociceptive thresholds returned to normal. These findings suggest that psychological stress is directly related to masticatory muscle pain. Removal of the stressor could be a useful method for relieving mechanical sensitivity increase induced by psychological stress.
\end{abstract}

\section{Introduction}

Currently, it is well accepted that stress induces analgesia. Studies have shown that acute stress increases nociceptive thresholds [1-4], leading to the possibility that stress, in general, produces stress-induced analgesia (SIA). However, more and more evidence in animal experiments suggests that repeated or prolonged stress can decrease nociceptive thresholds [1, 5-13]. For example, rats that were briefly exposed to short, emotionally arousing nonnoxious stress, such as holding or novel environments, displayed an immediate and transient hyperalgesia in response to thermal and electrical stimuli, which was followed by a longer period of analgesia [12]. In addition, prolonged stress from repeated exposure to a cold environment or restraint induces hyperalgesia $[5,7]$.

Exposure to a stressor in a laboratory generates a wide variety of adaptive responses, producing cellular, immune, endocrine, and behavioral effects, including changes in pain threshold [14]. Acute stress paradigms, in particular, can induce antinociceptive effects and produce the phenomenon of stress-induced analgesia (SIA) [15]. Unlike SIA, the mechanisms involved in stress-induced hyperalgesia $(\mathrm{SIH})$ are poorly understood. It was reported that chronic, repeated stress might activate the sympathoadrenal stress axis $[14,16-$ 19]. The sympathoadrenal stress axis mediates vagotomyinduced enhancement of bradykinin hyperalgesia [20].

The communication box method can produce psychological stress in animals, since they can perceive the responses of other animals exposed to physical stress delivered through an electric foot shock. This kind of intraspecies psychological stress was detected in earlier studies and further confirms that animals subjected to experimental anxiety within the communication box have increased stress hormones (plasma corticosterone level). Some animals also developed stomach ulcers [21]. The present study was designed to induce experimental psychological stress, such as anxiety, in rats using an emotional stress paradigm called intraspecies emotional communication within a communication box [22]. 
The hypothesis of this study was that psychological stress could induce changes in nociceptive thresholds, and an additional goal of this study was to determine whether nociceptive thresholds could return to normal after the stress is removed.

\section{Materials and Methods}

2.1. Animal Preparation. 48 male Sprague-Dawley rats, weighing from 160 to $180 \mathrm{~g}$ (approximately 35 days old) were housed in $80 \mathrm{~cm} \times 45 \mathrm{~cm} \times 40 \mathrm{~cm}$ cages in a temperaturecontrolled room at $24^{\circ} \mathrm{C}$ under a 12-hour light/dark cycle and were given free access to food and water. The rats were randomly divided into 4 groups: control group (CON; $n=8$ ), foot-shocked groups (further divided into three subgroups as FS-1, FS-2, and FS-3, with 8 rats in each subgroup), psychological stress group (PS; $n=8$ ), and drug treatment group (DT; $n=8)$.

2.2. Communication Box. The communication box [23] was selected as the psychological stress apparatus in this study. It consisted of 16 compartments which were each $16 \times$ $16 \mathrm{~cm}$ and were separated by transparent plastic boards with several small holes. The boards prevented each animal from physical contact but allowed them to receive cues such as visual, auditory, and olfactory sensations from the neighboring animals. Each compartment was equipped with a grid floor of stainless steel rods, $5 \mathrm{~mm}$ in diameter, placed at intervals of $0.3 \mathrm{~cm}$. An electric generator with 48 voltages (made by biology medical electron department of the Fourth Military Medical University, Xi'an, China) was connected to the grid floor to produce an electric current and generate an electric foot shock every 2 seconds. The grid floors of eight compartments were covered by plastic plates to prevent electric foot shock and served as non-foot-shock compartments for the PS rats (Figure 1).

The whole experiment contains 3 periods: prepsychological stress period, psychological stress period and psychological stress removal period. In the pre-psychological stress period, all rats were individually confined in each compartment of the communication box for one hour without any electric foot shock for one week in order to adapt them to the surroundings. This period lasted for 7 days. In the psychological stress period, two identical communication boxes were adopted, and the electric foot shocks were introduced to the FS-1 and FS-2 rats (stress senders) from 8:00 am to 9:00 am with foot shock daily. The PS and DT rats (stress responders) confined in the non-foot-shock compartments were then exposed to psychological stress cues from the neighboring FS rats, including shrieks, smells of urine or faeces, and jumping response. Consequently, the PS/DT rats were assumed to be in a state of fear or anxiety [24]. At the same time, FS-3 and CON rats were in a third communication box without foot shock delivery. This period lasted for 14 days, from 1 st day to 14 th day. In the psychological stress removal period (from 15th day to 28th day), procedure was similar to that of psychological stress period, but there was no foot shock delivered in all groups.

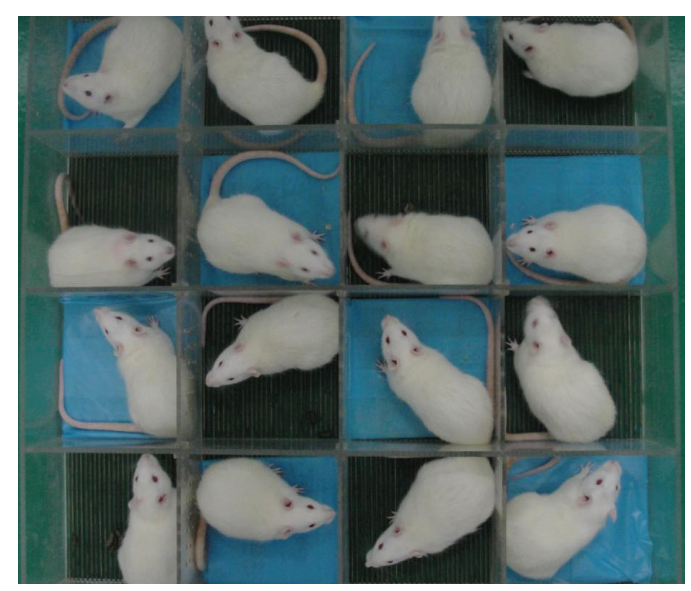

(a)

\begin{tabular}{|c|c|c|c|}
\hline PS/DT & FS & PS/DT & FS \\
\hline FS & PS/DT & FS & PS/DT \\
\hline PS/DT & FS & PS/DT & FS \\
\hline FS & PS/DT & FS & PS/DT \\
\hline
\end{tabular}

(b)

FIgure 1: The communication box used in this study. (a) Photo of the communication box with rats inside (PS refers to the grid floors covered by the blue plastic plates). (b) Schematic diagram of communication box (FS: foot-shocked group, PS: psychological stress group, DT: drug treatment group).

Diazepam was chosen to assess the effect of an anxiolytic drug on the masticatory muscle mechanical sensitivity of PS rats. Diazepam was dissolved in saline containing $40 \%$ propylene glycol. From Days 1 to 14 , diazepam $(1 \mathrm{mg} / \mathrm{kg})$ was injected subcutaneously into DT rats $30 \mathrm{~min}$ before the stress stimulation. The rats in PS/DT/CON groups were weighed weekly in the experiment. The behaviors were immediately evaluated by an elevated plus-maze apparatus after stress. The experimental procedures were reviewed and approved by the Ethics Committee of the Fourth Military Medical University. The FS (1, 2, and 3) rats were only used to induce psychological stress in the neighboring PS/DT rats and were not included in the following investigations.

2.3. Elevated Plus-Maze Tests. The elevated plus-maze (EPM) apparatus (RD1208, Shanghai Mobiledatum Corporation, Shanghai, China) consists of two open arms $(50 \times 10 \mathrm{~cm})$ and two enclosed arms $(50 \times 10 \mathrm{~cm}$, with $50-\mathrm{cm}$ high walls $)$ extending from a central square platform $(10 \times 10 \mathrm{~cm})$. The same types of arm were arranged in the opposite position. The apparatus was elevated $50 \mathrm{~cm}$ above the floor in a quiet 
room with the temperature controlled at $20^{\circ} \mathrm{C}$. The light in the room was just bright enough to clearly observe the rats' movement within a 1.5 meter range. Two of the opposing arms $(50 \mathrm{~cm} \times 10 \mathrm{~cm})$ were enclosed by $40 \mathrm{~cm}$ high side and end walls (enclosed arms). The other two arms did not have walls (open arms). At the beginning of the experiment, the animals were placed in the central area $(10 \mathrm{~cm} \times 10 \mathrm{~cm})$ of the maze facing an enclosed arm. The exposure lasted for five minutes and certain parameters were recorded. Entry into one arm was recorded when an animal placed all four paws past the line dividing the central square from the open arms. The test arena was wiped with a damp cloth after each trial. The number of entries into the open/closed arms and the time spent in open arms/closed arms were measured by an observer who was blinded to the treatment conditions of the animals. Then the percent of open arm entries $(100 \times$ open/open + enclosed entries, $\mathrm{OE} \%)$ and the time spent in the open arms $(100 \times$ open/open + enclosed arm time, OT\%) of the EPM were calculated for each rat. We used OE\% and OT\% to analyze the anxiety level of each animal [25-28].

2.4. Method for Assessing Mechanical Sensitivity. Instead of standing on meshed metal or a grid surface, the rats stood on a soft pad. Habituation required no more than petting the rats, and it was completed within half an hour. The smell that developed on the glove of the handler by handling the animals appeared to facilitate the habituation [29]. This test environment was advantageous in that the rats were not restrained, but their movements were still restricted. The animals were habituated prior to testing. The testing was similar to a procedure described by Ren [29]. Von Frey filaments were used to assess the muscle mechanical sensitivity. In our research, two orofacial areas were tested: the temporal muscle region at the central point of the line between the orbit and the tragus and the masseter muscle belly region, at a site $10 \mathrm{~mm}$ inferior to the temporal muscle testing point (Figure 2). At these locations, muscular contractions could be palpated during mastication. During testing, force was applied with a probe-oriented perpendicular to the sagittal plane.

Head flinching, characterized as sudden quick head withdrawal, or vocalization/crying, was considered to be positive pain responses. The response threshold was defined as the lowest bending force of the filaments that produced at least three positive responses in five trials with $5 \mathrm{~s}$ interval. An ascending stiffness series of the filaments was used for the test. The bending force of the Von Frey filaments was verified on a balance with a resolution up to $0.001 \mathrm{~g}$ before test (Ohaus, Model GT410D) (Table 1). For this study, we defined increased mechanical sensitivity as a statistically significant decreased withdrawal threshold compared to baseline, which was obtained in "pre-psychological stress period".

From seven days before applying the emotional stressor to the fourteenth day after psychological stress removal, we recorded the mechanical threshold of the masticatory muscle. We used these indexes to compare changes in the degree of mechanical pain before and after psychological stress. The

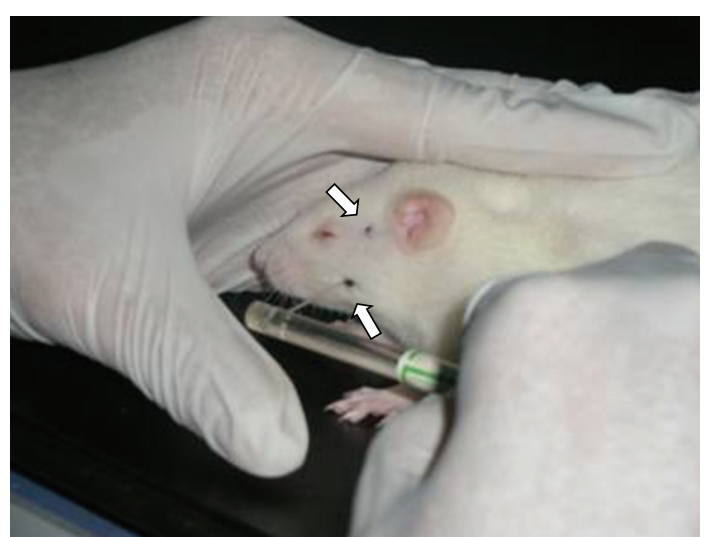

Figure 2: Photograph illustrating the method for assessing mechanical nociceptive thresholds in the rat. The experimenter's hand provided a comfortable "rest" for the rat. Then a von Frey monofilament was probed against the orofacial region. Note that the rat was unrestrained and stayed in one position. The arrows indicate where the tip of the monofilament was probing against the rat's skin.

rats were tested daily half an hour after psychological stress was applied. For all of the data reported here, the observer was blinded to the treatment conditions for the rats.

2.5. Statistical Analysis. Experimental data were analyzed by a one-way analysis of variance (ANOVA) across the CON group, PS group, and the DT group using SPSS, version 11.0 (SPSS Co., Chicago, Illinois). The SNK-q test was also used to calculate any differences between the two groups. A $P$-value less than .05 was considered to be statistically significant.

\section{Results}

The purpose of this study was to explore the effects of psychological stress upon sensitivity of the masticatory muscles. But the foot-shocked group rats experienced a great part of physical stress, so we did not include this group in the experimental evaluations.

Body weights were measured during the exposure to psychological stress (Figure 3). Initially, the mean body weight of rats did not differ between the PS, DT, and CON groups $(170.8 \pm 1.7 \mathrm{~g}, 175.0 \pm 2.3 \mathrm{~g}$, and $173 \pm 3.2 \mathrm{~g}$, resp., $P>$.05). There was no significant difference in the body weight gain between the PS, DT and CON groups (320.7 \pm $7.1,323.2 \pm 5.7$, and $325.2 \pm 4.8$, resp., $P>.05$ ) during the whole study, which contained a psychological stress period and a removal of stress period.

The anxiety behaviors of rats were measured by $\mathrm{OE} \%$ and OT\% in EPM. As shown in Figure 4(a), the baseline measurements did not show significant differences among the control $(48.13 \pm 1.47 \%)$, DT $(47.56 \pm 1.79 \%)$ and PS $(46.53 \pm 1.41 \%)$ groups $(P>.05)$. During the psychological stress period, on 1st day, 7th day, and 14th day, PS rats had a smaller percentage (\%) of entries into the open arms (open arm entry, OE) $(42.15 \pm 1.01 \%, 34.03 \pm 1.19 \%, 38.53 \pm$ $1.27 \%$, resp.) compared to rats in the CON group ( $49.25 \pm$ $1.89 \%, 49 \pm 1.42 \%, 49.38 \pm 1.42 \%$, resp. $)(P<.05)$. The data 
TABLE 1: Forces corresponding to the Von Frey filaments used in the tests.

\begin{tabular}{lccc}
\hline Ordinal no. & $\begin{array}{c}\text { Calibrated force } \\
(\text { mean gram } \pm \text { SEM) } \\
(\mathrm{g})\end{array}$ & Ordinal no. & $\begin{array}{c}\text { Calibrated force } \\
(\text { mean gram } \pm \text { SEM }) \\
(\mathrm{g})\end{array}$ \\
\hline 1 & $1.36 \pm 0.011$ & 9.3 & $79.3 \pm 0.93$ \\
2 & $3.24 \pm 0.033$ & 9.4 & $83.7 \pm 1.05$ \\
3 & $4.34 \pm 0.027$ & 9.5 & $89.5 \pm 1.23$ \\
4 & $5.60 \pm 0.113$ & 9.6 & $95.1 \pm 1.34$ \\
5 & $6.51 \pm 0.031$ & 9.7 & $101.3 \pm 1.45$ \\
6 & $11.0 \pm 0.07$ & 9.8 & $107.3 \pm 1.66$ \\
7 & $15.0 \pm 0.08$ & 9.9 & $112.3 \pm 1.78$ \\
8 & $25.6 \pm 0.43$ & 10 & $118 \pm 2$ \\
9 & $62.9 \pm 0.71$ & 10.1 & $125 \pm 1.75$ \\
9.1 & $68.2 \pm 0.77$ & 10.2 & $130 \pm 2.25$ \\
9.2 & $74.1 \pm 0.86$ & 10.3 & $136 \pm 2.41$ \\
\hline
\end{tabular}

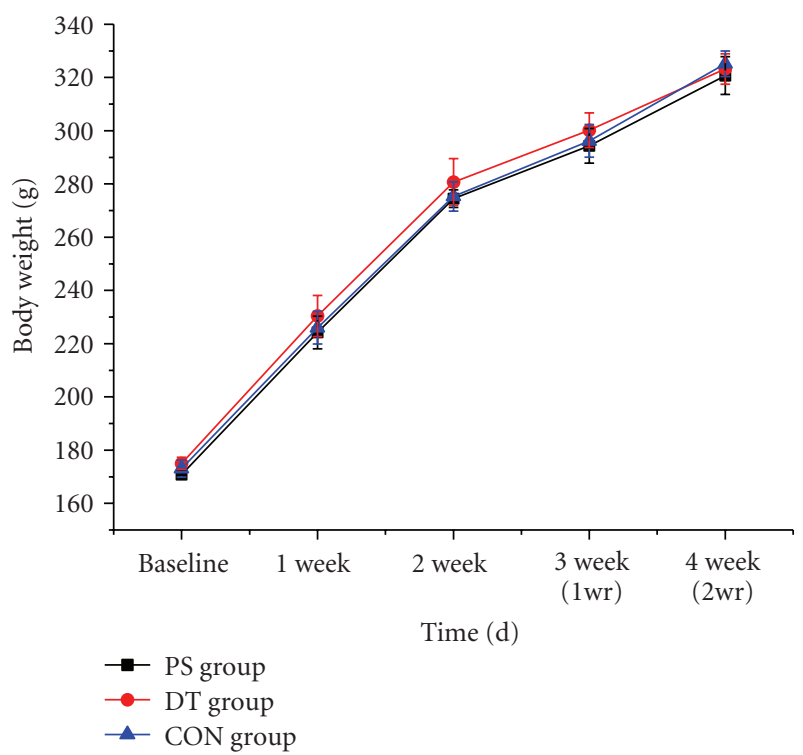

FIGURE 3: Body weights of the rats. There was no significant difference in the body weight gain between the PS, DT and CON groups. The time point of one week after psychological stress removal is indicated by " 1 week after removal of psychological stress ( $1 \mathrm{wr})$." The time point two weeks after psychological stress removal is indicated by " 2 weeks after removal of psychological stress ( $2 \mathrm{wr})$ ".

of DT rats $(46.53 \pm 0.71 \%, 46.9 \pm 0.66 \%, 47.65 \pm 1.06 \%$, resp.) were between the PS and CON group, with significant differences compared to both of them $(P<.05)$. As shown in Figure 4(b), the baseline measurements did not show significant differences among the control $(67.75 \pm 1.29 \%)$, DT $(67.19 \pm 1.12 \%)$ and PS $(66.53 \pm 1.41 \%)$ groups $(P>$ $.05)$. During the psychological stress period, on 1st day, 7 th day and 14 th day, the PS $(62.06 \pm 1.40 \%, 55.31 \pm 1.48 \%$, $58.59 \pm 1.24 \%$, resp. $)$ and DT $(64.78 \pm 1.40 \%, 65.05 \pm$
$1.58 \%, 67.6 \pm 1.34 \%$, resp.) rats preferred to spend a smaller percentage of time in the open arms (OT\%) compared to the CON group rats $(68.5 \pm 1.19 \%, 67.88 \pm 1.43 \%, 69.49 \pm$ $0.99 \%$, resp. $)(P<.05)$, and the data in DT rats were higher than those of PS rats $(P<.05)$. The decreasing percentages of $\mathrm{OE}$ and OT indicated that the rats in the PS and DT groups were mentally under tension, with the PS rats experiencing more severe anxiety. In the psychological stress removal period, $\mathrm{OE} \%$ and OT\% in PS $(44.02 \pm 2.1 \%, 63.18 \pm 1.23 \%)$ group were lower than DT $(48.19 \pm 1.5 \%, 67.1 \pm 1.05 \%)$ and CON group $(48.88 \pm 1.74 \%, 67.79 \pm 1.17 \%)$ on 21 st day $(P<.05)$, and they $(48.55 \pm 1.85 \%, 66.65 \pm 1.83 \%)$ returned to normal with no differences to the DT $(49.33 \pm$ $2.08 \%, 67.6 \pm 1.91 \%)$ and CON $(49.64 \pm 1.36 \%, 68.23 \pm$ $0.97 \%)$ groups on 28 th day $(P>.05)$.

In the prepsychological stress period, the mechanical thresholds of the bilateral masseter and the temporal muscles were assessed by Von Frey filaments. The head withdrawal threshold gradually increased then stabilized on the 7th day (shown as the pre-stress stage of Figures 5(a)-5(d)). It meant that the mechanical sensitivity decreased to a stable level as "baseline" used in psychological stress period.

Increased mechanical sensitivity was induced in both the temporal muscles and the masseter muscles on both sides of the head following psychological stress (shown as the onstress stage of Figures 5(a)-5(d)). During the psychological stress period, increased mechanical sensitivity of the PS rats was observed in the bilateral masseter and temporal muscles with the peak time on the 7th day. Then the mechanical sensitivity was alleviated during the following days till the 14th day at a pain threshold lower than the CON rats $(P<.05)$. There were no significant differences between bilateral masseter muscles and temporal muscles $(P>.05)$. After removing the psychological stress, the mechanical pain threshold of temporal muscles in PS group gradually elevated and returned to normal on the 28th day $(P>.05)$. At 


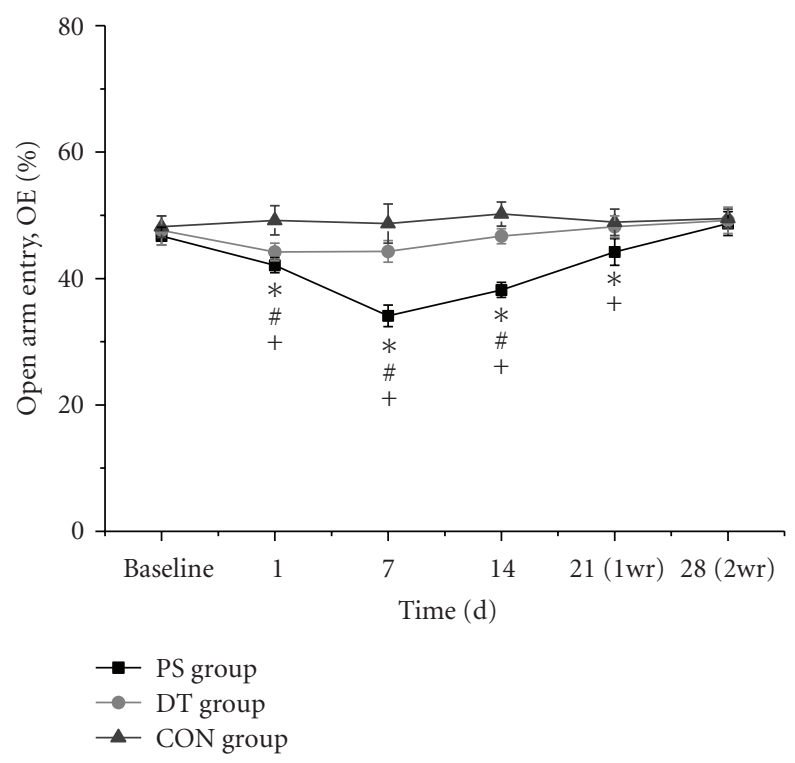

(a)

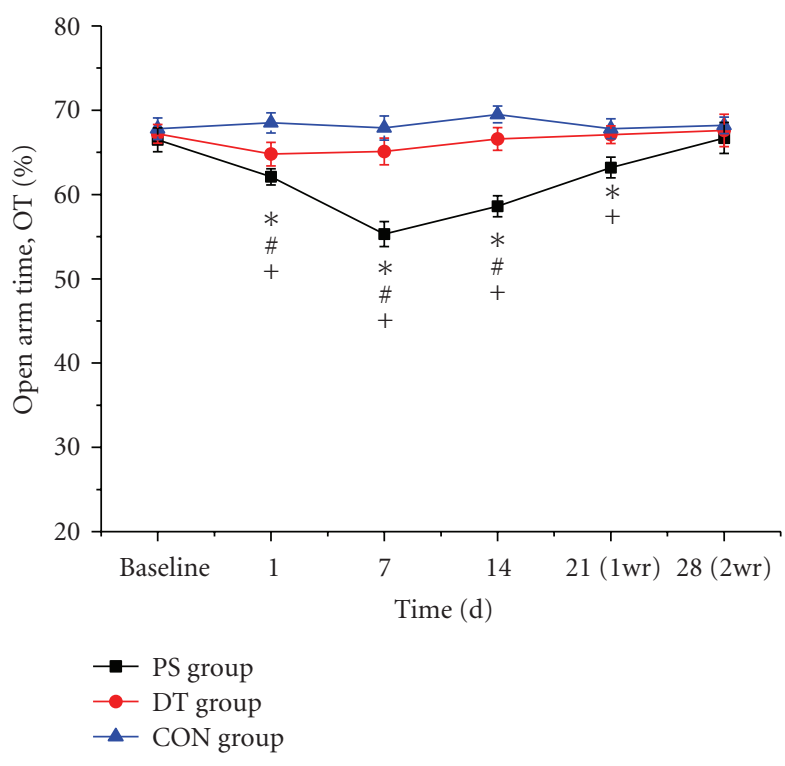

(b)

Figure 4: Comparison of the percentage of open arm entries (OE\%) (a) and the percentage of time spent in the open arms (OT\%) (b) in the elevated plus-maze test among groups. $\left({ }^{*} P<.05\right.$, PS group versus $\mathrm{CON}$ group; ${ }^{+} P<.05$, PS group versus DT group; ${ }^{\#} P<.05$, DT group versus CON group).

the same time, the mechanical sensitivity of masseter muscles in PS group was gradually alleviated and finally returned to the baseline level on the 28th day $(P>.05)$ (shown as the post-stress stage of Figures $5(\mathrm{a})-5(\mathrm{~d}))$. Through the whole experiment, the PS group showed greater mechanical sensitivity than the CON and DT groups $(P<.05)$, but no significant difference was detected between the CON group and the DT group $(P>.05)$.

\section{Discussion}

The communication box method can produce anxiety in animals as they perceive the responses of other animals exposed to physical stress from an electric foot shock [23]. In the present study, the communication box was used to simulate a psychologically stressful environment and to determine whether anxiety would induce increased mechanical sensitivity in the masticatory muscles. We also examined whether the nociceptive response could return to normal after the stressor was removed.

It is interesting that when the rats were first placed into the narrow chart of communication box which meant new environment to them, they were already under psychological stress actually. So at the beginning of this period, the increased mechanical sensitivity already existed. As the time went on, the mechanical sensitivity decreased to a stable level considered as "baseline," which was used for comparison with psychological stress period. This may indicate that the PS group had learned to accommodate themselves to the stressful environment of communication box without foot shock delivered.
The stress stimulation was commenced in the psychological stress period. From the very first day with stress, decreased head withdrawal thresholds induced by anxiety were observed in the PS group, but not in the DT and CON groups, which may indicate that psychological stress stimuli upregulated the mechanical sensitivity of masticatory muscles. Then the mechanical sensitivity was alleviated from 8 to 14 days in the PS group. This may indicate that the PS rats had learned to accommodate to the stressful environment. This was consistent with the results of Ye Cao's research [30]. They found that increased mechanical thresholds (decreased head withdrawal threshold to mechanical pressure) were induced in both the temporal muscles and the masseter muscles on both sides of the head following occlusal interference. The mechanical threshold decreased from the first day and peaked on the seventh day. Besides, the present results showed that no increased mechanical sensitivity occurred from 0 to 14 days in the CON group, which indicated that this group did not experience psychological stress during the whole period. Meanwhile, there was also no evidence of increased mechanical sensitivity in the DT group. It might be attributed to the gradual blocking effect of diazepam, which has the strong potency of suppressing anxiety. In the results of $\mathrm{OE} \%$ and OT\% in EPM, we found that the data in DT rats was lower than CON rats, but higher than PS rats. It may be due to low doses of diazepam used in our experiment, which could not completely block stress stimulator. We noticed that the results of mechanical test and EPM tests were not entirely consistent, which might be caused by subjective error of the experimenter or some unknown factors and still need further study.

In the psychological stress removal period, without any foot shock, the increased mechanical sensitivity of PS rats 


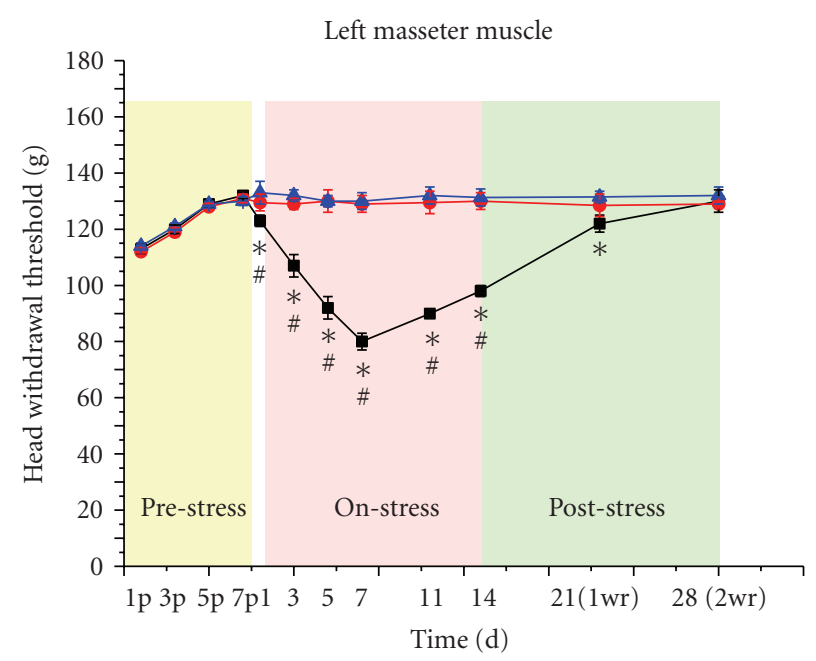

(a)

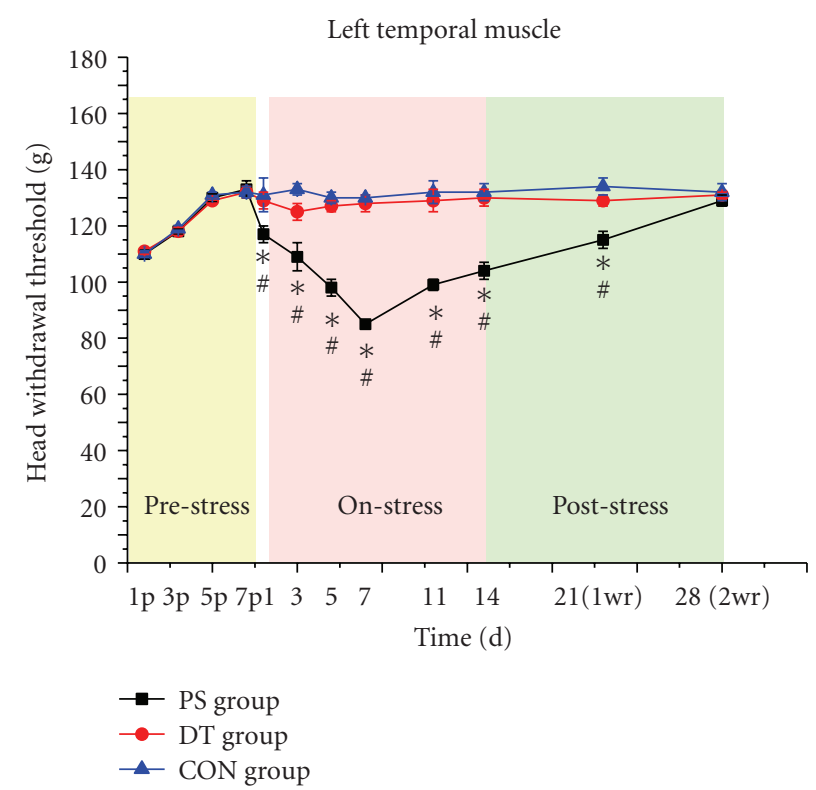

(c)

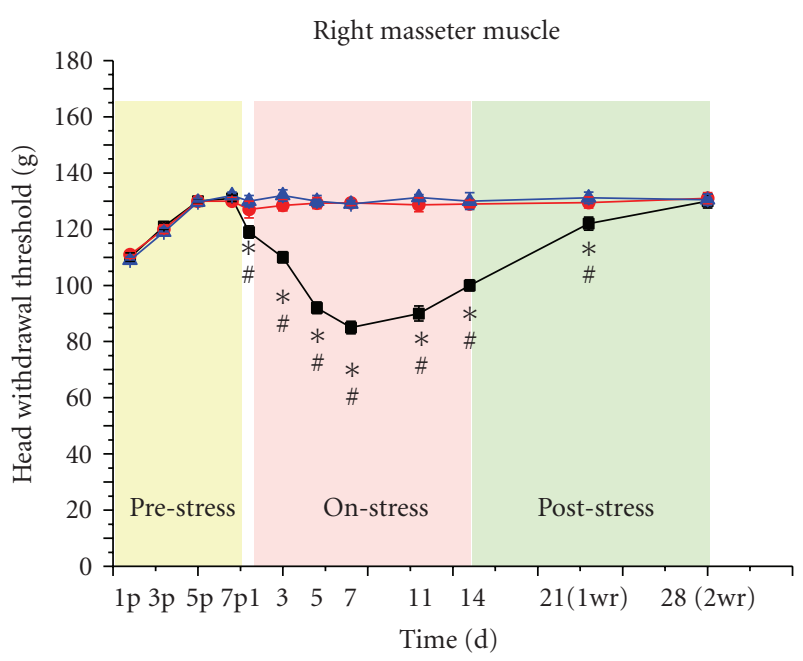

(b)

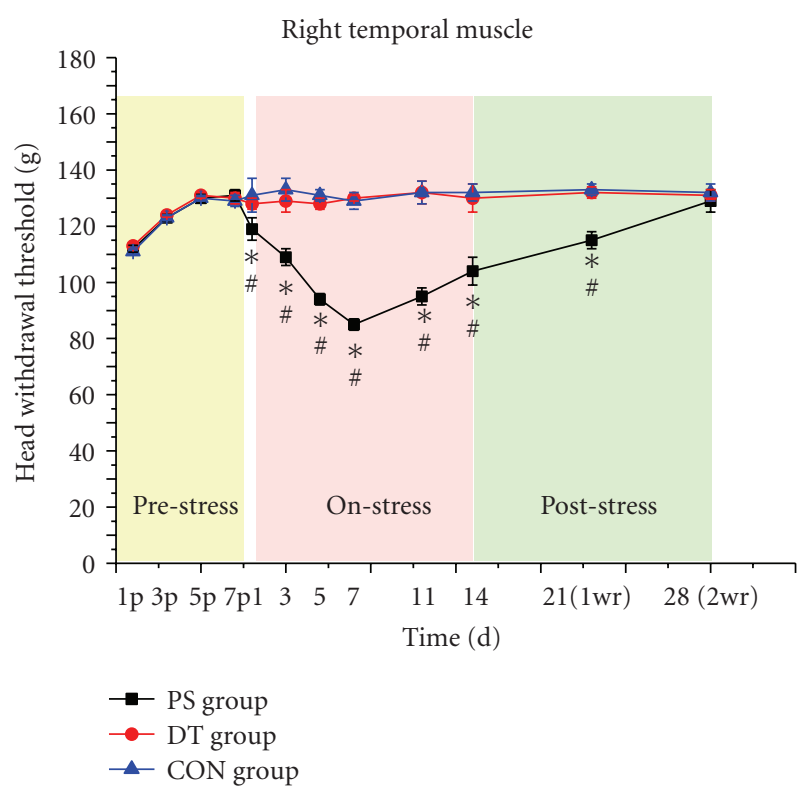

(d)

FIGURE 5: The time course of changes in the head withdrawal threshold to bilateral stimulation of masseter ((a), (b)) and temporal muscles ((c), (d)) during pre-psychological stress period, psychological stress, and psychological stress removal period. During the pre-psychological stress period, the head withdrawal threshold gradually increased then stabilized on the 7th day (yellow-shadowed part in Figures 5(a)-5(d)). During the psychological stress period, the mechanical nociceptive threshold started to decrease on day 1 after psychological stress, and reached lowest on days 5-7 then started to increase again in the following days till the 14th day (pink-shadowed part in Figures 5(a)-5(d)). After removal of psychological stress, the index of each group gradually diminished and the pain threshold returned to normal on the 28th day. No significant difference was found between CON group and DT group (cyan-shadowed part in Figures $5($ a $)-5(d))$. $\left({ }^{*} P<.05\right.$, PS group versus CON group; ${ }^{\#} P<.05$, PS group versus DT group; the letter "P" in the horizontal axis meant pre-psychological stress stage.)

was alleviated from the 15th day and decreased until 28th day without any significant differences to CON rats. This trend suggested that removing the stressor would be an effective means for curing diseases induced by anxiety. Meanwhile, the analgesic response to acute stress was not observed in this study. It suggested that, in this model of chronic stress, the reinstatement of the activity of systems involved in the nociceptive response has different patterns, as shown by the period of time and the types of response that were evaluated (the basal measurement or the nociceptive response to acute stress). Prolonged stress could lead to more lasting alterations in the neural systems involved in nociception modulation.

Changes in the weight of the thymus, spleen and adrenal glands are usually found when stress is induced [31]. Ishikawa [21] also observed no changes in the weight of organs or overall body weight in rats exposed to emotional stress. The present study showed that there was no significant difference between the PS, DT, and CON groups, which is 
consistent with the results of Rosales's study [23]. These results may indicate that the effect of the anxiety induced by the communication box was not strong enough to induce a weight gain, but it was sufficient to induce increased mechanical sensitivity in masticatory muscle.

Unlike stress-induced analgesia, the mechanisms in volved in stress-induced mechanical sensitivity increase are not well known. Satoh suggested that long-lasting mechanical hyperalgesia (3 days) induced by prolonged cold stress involved peptide-containing primary afferents (substance-P and calcitonin-gene-related peptide) [5]. Quintero suggested that increased thermal and chemical nociception (8-9 days) observed after a subchronic swimming stress might be mediated by changes in the activity of the central serotonergic system [32]. Works in human showed that a reduction in the pain threshold after long-term psychoemotional stress was probably due to a reduction in the activity of the brain's opioid system [33]. Preliminary investigations have also found that psychological stress, such as anxiety and tension, could increase the myoelectricity $[34,35]$ and parafunction activities of the masticatory muscle [23].

Stress-provoking stimuli are known to activate the dopaminergic system [36]. According to research by Ida et al. [37], the mesoprefrontal dopamine system plays an important role in the control of negative states such as fear and/or anxiety. Diazepam has been suggested to induce inhibitory effects on the activation of mesoprefrontal dopamine neurons and the hypothalamic-pituitary-adrenocortical axis [38] The drug also has been used as an antianxiety drug in several stress-related experiments using the communication box [23, 37]. Throughout the experiment in the current study, the PS group showed greater mechanical sensitivity than the CON and DT groups, but no significant difference was detected between the CON group and the DT group. This result is consistent with Wood et al. [39]. They found that the supplementation of serotonergic tone during subchronic stress exposure appears to provide prophylaxis for stress-induced mechanical sensitivity, but this effect does not obviate a role for dopamine. In fact, even though there is a disruption in both the serotonergic and dopaminergic function that occurs within the nucleus accumbens (NAc) following chronic stress, the impact on dopamine outlasts the impact on serotonin (5-HT). This difference may be responsible for the persistent expression of stress-induced hyperalgesia after serotonergic function has been normalized [39]. Secondly, from the therapeutic implications, benzodiazepines (e.g., diazepam as used in the present experiment) are pharmacologically linked to GABAergic (gamma aminobutyric acid) neurotransmission. On administering diazepam, the benzodiazepine receptors are activated and, since they are allosterically linked to GABA-A receptors, they modulate the chloride channel opening indirectly and induce inhibition of that neuronal function [23].

\section{Conclusions}

The experiments in this study indicated that anxiety could induce increased mechanical sensitivity through exposure to repeated stress in rats. We also demonstrated that changes in withdrawal thresholds to mechanical pressure could be blocked by diazepam in repeatedly stressed rats. Further studies concerning the mechanisms of stress-induced mechanical sensitivity would be relevant to studies of the etiology of chronic pain disorders.

\section{Acknowledgment}

This study was financially supported by National Natural Science Foundation of China (nos. 30672326, 81070851). F. Huang and M. Zhang contributed equally to the work.

\section{References}

[1] E. Jorum, "Analgesia or hyperalgesia following stress correlates with emotional behavior in rats," Pain, vol. 32, no. 3, pp. 341$348,1988$.

[2] S. Mousa, C. H. Miller Jr., and D. Couri, "Corticosteroid modulation and stress-induced analgesia in rats," Neuroendocrinology, vol. 33, no. 5, pp. 317-319, 1981.

[3] J. C. Willer, H. Dehen, and J. Cambier, "Stress-induced analgesia in humans: endogenous opioids and naloxonereversible depression of pain reflexes," Science, vol. 212, no. 4495, pp. 689-691, 1981.

[4] E. Widy-Tyszkiewicz, P. Mierzejewski, M. Kohutnicka, and A. Czlonkowski, "Cold water stress induced analgesia in unilateral inflammation of the hindpaw in hypertensive and normotensive rats," Polish Journal of Pharmacology, vol. 47, no. 4, pp. 313-320, 1995.

[5] M. Satoh, Y. Kuraishi, and M. Kawamura, "Effects of intrathecal antibodies to substance $\mathrm{P}$, calcitonin gene-related peptide and galanin on repeated cold stress-induced hyperalgesia: comparison with carrageenan-induced hyperalgesia," Pain, vol. 49, no. 2, pp. 273-278, 1992.

[6] S. Bradesi, I. Schwetz, H. S. Ennes et al., "Repeated exposure to water avoidance stress in rats: a new model for sustained visceral hyperalgesia," American Journal of Physiology, vol. 289, no. 1, pp. G42-G53, 2005.

[7] G. H. Gameiro, P. H. Gameiro, A. da Silva Andrade et al., "Nociception- and anxiety-like behavior in rats submitted to different periods of restraint stress," Physiology and Behavior, vol. 87, no. 4, pp. 643-649, 2006.

[8] S. G. Khasar, P. G. Green, and J. D. Levine, "Repeated sound stress enhances inflammatory pain in the rat," Pain, vol. 116, no. 1-2, pp. 79-86, 2005.

[9] I. L. da Silva Torres, C. D. Bonan, L. Crema et al., "Effect of drugs active at adenosine receptors upon chronic stressinduced hyperalgesia in rats," European Journal of Pharmacology, vol. 481, no. 2-3, pp. 197-201, 2003.

[10] I. L. da Silva Torres, S. N. S. Cucco, M. Bassani et al., "Longlasting delayed hyperalgesia after chronic restraint stress in rats-effect of morphine administration," Neuroscience Research, vol. 45, no. 3, pp. 277-283, 2003.

[11] E. Jorum, "Noradrenergic mechanisms in mediation of stressinduced hyperalgesia in rats," Pain, vol. 32, no. 3, pp. 349-355, 1988.

[12] C. Vidal and J. Jacob, "Hyperalgesia induced by non-noxious stress in the rat," Neuroscience Letters, vol. 32, no. 1, pp. 75-80, 1982.

[13] C. Vidal and J. Jacob, "Hyperalgesia induced by emotional stress in the rat: an experimental animal model of human 
anxiogenic hyperalgesia," Annals of the New York Academy of Sciences, vol. 467, pp. 73-81, 1986.

[14] K. Pacák and M. Palkovits, "Stressor specificity of central neuroendocrine responses: implications for stress-related disorders," Endocrine Reviews, vol. 22, no. 4, pp. 502-548, 2001.

[15] J. W. Lewis, J. T. Cannon, and J. C. Liebeskind, "Opioid and nonopioid mechanisms of stress analgesia," Science, vol. 208, no. 4444, pp. 623-625, 1980.

[16] P. H. Black, "Stress and the inflammatory response: a review of neurogenic inflammation," Brain, Behavior, and Immunity, vol. 16, no. 6, pp. 622-653, 2002.

[17] P. H. Black and L. D. Garbutt, "Stress, inflammation and cardiovascular disease," Journal of Psychosomatic Research, vol. 52, no. 1, pp. 1-23, 2002.

[18] P. H. Black, "The inflammatory response is an integral part of the stress response: implications for atherosclerosis, insulin resistance, type II diabetes and metabolic syndrome X," Brain, Behavior, and Immunity, vol. 17, no. 5, pp. 350-364, 2003.

[19] K. Pacak, M. Palkovits, I. J. Kopin, and D. S. Goldstein, "Stressinduced norepinephrine release in the hypothalamic paraventricular nucleus and pituitary-adrenocortical and sympathoadrenal activity: in vivo microdialysis studies," Frontiers in Neuroendocrinology, vol. 16, no. 2, pp. 89-150, 1995.

[20] S. G. Khasar, F. J. P. Miao, W. Jänig, and J. D. Levine, "Vagotomy-induced enhancement of mechanical hyperalgesia in the rat is sympathoadrenal-mediated," Journal of Neuroscience, vol. 18, no. 8, pp. 3043-3049, 1998.

[21] M. Ishikawa, C. Hara, S. Ohdo, and N. Ogawa, "Plasma corticosterone response of rats with sociopsychological stress in the communication box," Physiology and Behavior, vol. 52, no. 3, pp. 475-480, 1992.

[22] T. List, K. Wahlund, and B. Larsson, "Psychosocial functioning and dental factors in adolescents with temporomandibular disorders: a case-control study," Journal of Orofacial Pain, vol. 15, no. 3, pp. 218-227, 2001.

[23] V. P. Rosales, K. Ikeda, KO. Hizaki, T. Naruo, S. I. Nozoe, and G. Ito, "Emotional stress and brux-like activity of the masseter muscle in rats," European Journal of Orthodontics, vol. 24, no. 1, pp. 107-117, 2002.

[24] N. Ogawa, C. Hara, and M. Ishikawa, "Characteristics of socio-psychological stress induced by the communication box method in the mice and rats," in Environmental Stress, O. Manninen, Ed., pp. 417-427, ACES Publishing, Tampele, 1990.

[25] S. Pellow, P. Chopin, S. E. File, and M. Briley, "Validation of open: closed arm entries in an elevated plus-maze as a measure of anxiety in the rat," Journal of Neuroscience Methods, vol. 14, no. 3, pp. 149-167, 1985.

[26] R. G. Lister, "The use of a plus-maze to measure anxiety in the mouse," Psychopharmacology, vol. 92, no. 2, pp. 180-185, 1987.

[27] G. Biała and M. Kruk, "Amphetamine-induced anxiety-related behavior in animal models," Pharmacological Reports, vol. 59, no. 6, pp. 636-644, 2007.

[28] M. Rabbani, S. E. Sajjadi, and A. Mohammadi, "Evaluation of the anxiolytic effect of Nepeta persica Boiss. in mice," Evidence-Based Complementary and Alternative Medicine, vol. 5, no. 2, pp. 181-186, 2008.

[29] K. Ren, "An improved method for assessing mechanical allodynia in the rat," Physiology and Behavior, vol. 67, no. 5, pp. 711-716, 1999.

[30] Y. Cao, Q. F. Xie, K. Li, A. R. Light, and K. Y. Fu, “Experimental occlusal interference induces long-term masticatory muscle hyperalgesia in rats," Pain, vol. 144, no. 3, pp. 287-293, 2009.
[31] J. T. Marsh and A. F. Rasmussen Jr., "Response of adrenals, thymus, spleen and leucocytes to shuttle box and confinement stress," Proceedings of the Society for Experimental Biology and Medicine, vol. 104, pp. 180-183, 1960.

[32] L. Quintero, M. Moreno, C. Avila, J. Arcaya, W. Maixner, and H. Suarez-Roca, "Long-lasting delayed hyperalgesia after subchronic swim stress," Pharmacology Biochemistry and Behavior, vol. 67, no. 3, pp. 449-458, 2000.

[33] I. Y. Ashkinazi and E. A. Vershinina, "Pain sensitivity in chronic psychoemotional stress in humans," Neuroscience and Behavioral Physiology, vol. 29, no. 3, pp. 333-337, 1999.

[34] R. A. Nicholson, D. R. Townsend, and S. E. Gramling, "Influence of a scheduled-waiting task on EMG reactivity and oral habits among facial pain patients and no-pain controls," Applied Psychophysiology Biofeedback, vol. 25, no. 4, pp. 203219, 2000.

[35] C. M. Tsai, S. L. Chou, E. N. Gale, and W. D. McCall Jr., "Human masticatory muscle activity and jaw position under experimental stress," Journal of Oral Rehabilitation, vol. 29, no. 1, pp. 44-51, 2002.

[36] E. L. Bliss and J. Ailion, "Relationship of stress and activity to brain dopamine and homovanillic acid," Life Sciences, vol. 10, no. 20, pp. 1161-1169, 1971.

[37] Y. Ida, A. Tsuda, K. Sueyoshi, I. Shirao, and M. Tanaka, "Blockade by diazepam of conditioned fear-induced activation of rat mesoprefrontal dopamine neurons," Pharmacology Biochemistry and Behavior, vol. 33, no. 2, pp. 477-479, 1989.

[38] H. Kaneyuki, H. Yokoo, A. Tsuda et al., "Psychological stress increases dopamine turnover selectively in mesoprefrontal dopamine neurons of rats: reversal by diazepam," Brain Research, vol. 557, no. 1-2, pp. 154-161, 1991.

[39] P. B. Wood, "Stress and dopamine: Implications for the pathophysiology of chronic widespread pain," Medical Hypotheses, vol. 62, no. 3, pp. 420-424, 2004. 


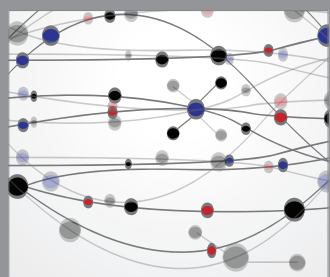

The Scientific World Journal
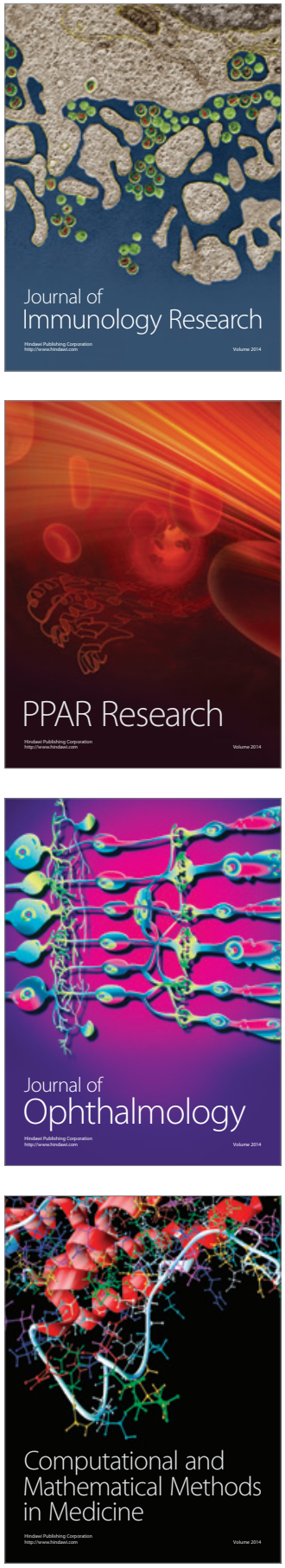

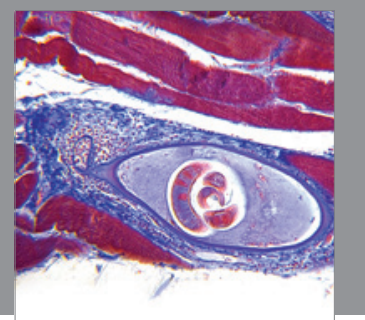

Gastroenterology

Research and Practice
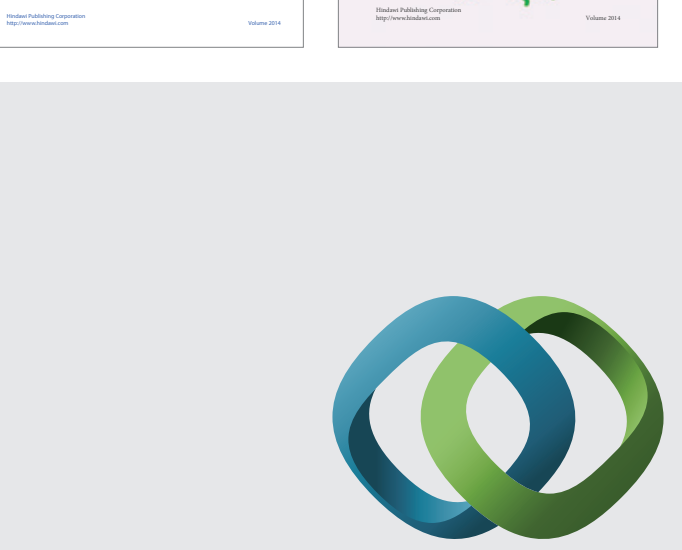

\section{Hindawi}

Submit your manuscripts at

http://www.hindawi.com
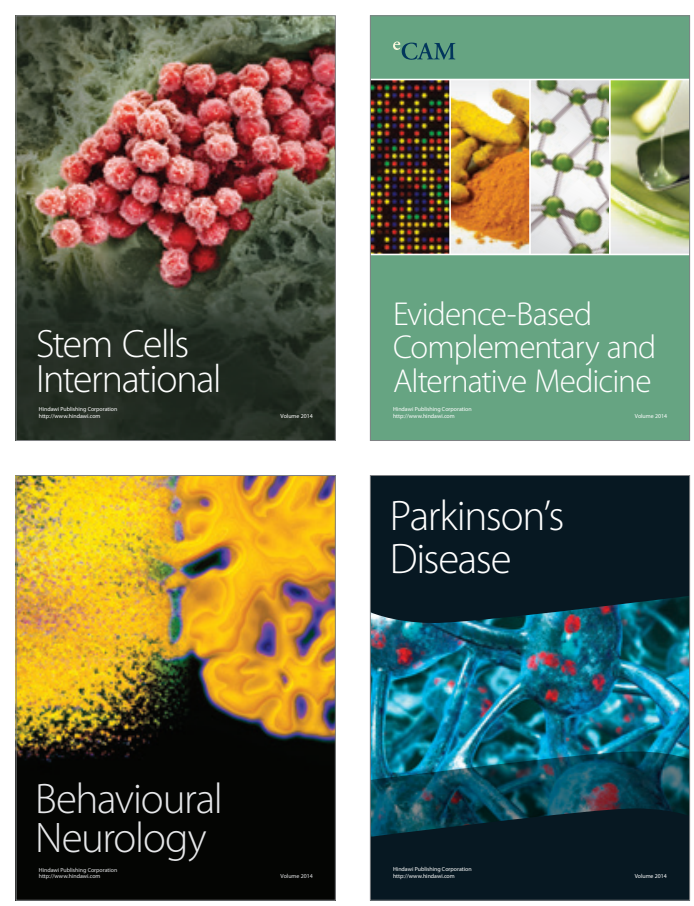

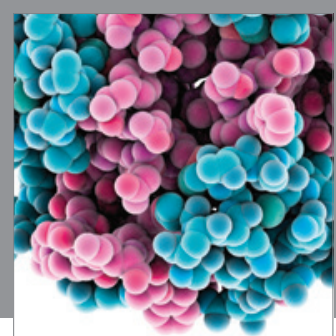

Journal of
Diabetes Research

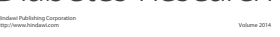

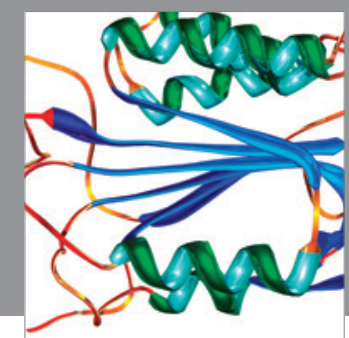

Disease Markers
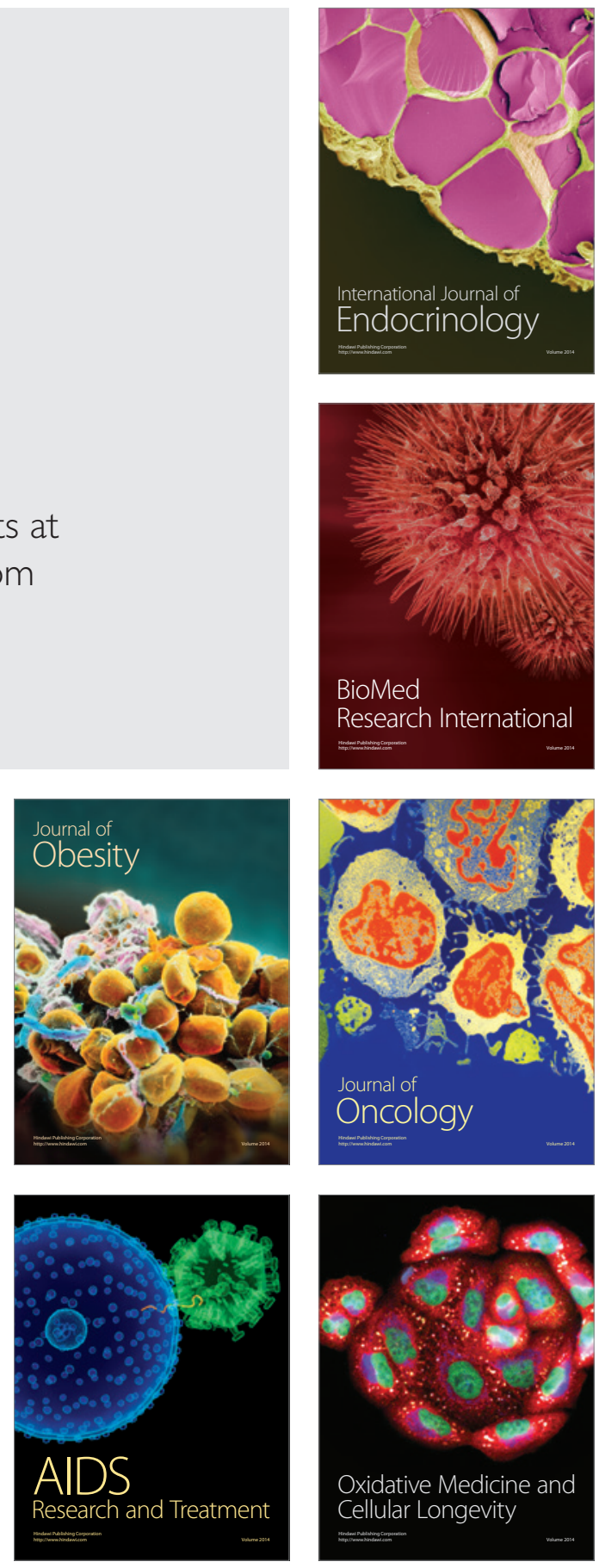